New Perspectives 2020 (2.0)

Fermilab, USA

\title{
Radiative corrections to
}

\section{CCQE neutrino-nucleon scattering in effective field theory}

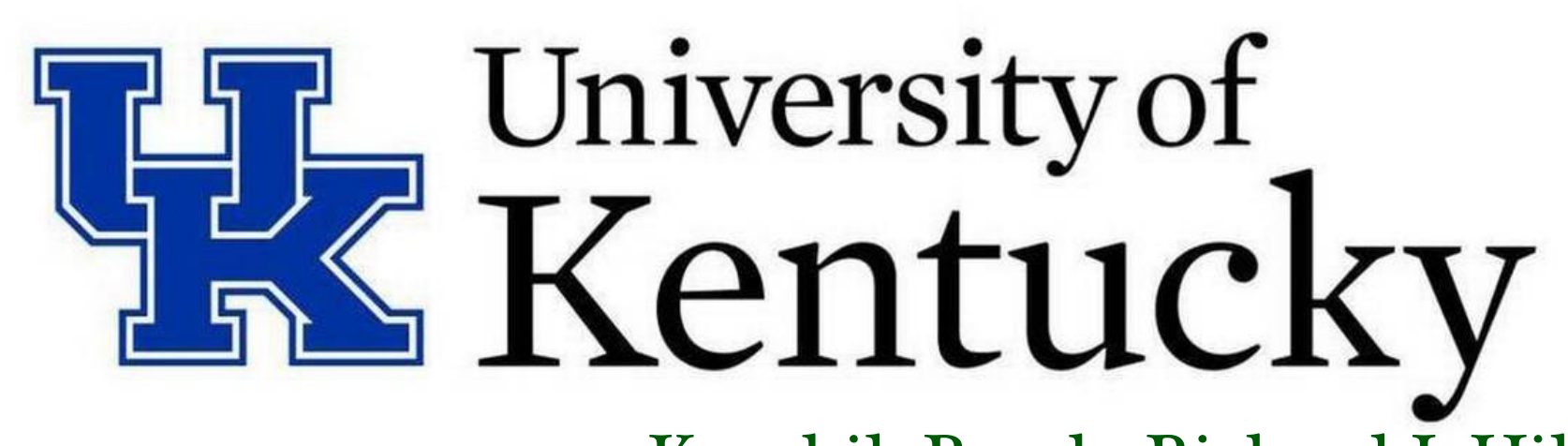

\section{Oleksandr Tomalak}

Kaushik Borah, Richard J. Hill, Gabriel Lee and O. T. (arXiv: 2003.13640)

O. T. (arXiv: 2008.03527)

Qing Chen, Richard J. Hill, Kevin S. McFarland and O. T. (arXiv: to appear) 


\section{CCQE. Why should we care?}

- neutrino-nucleus cross sections and future accelerator-based fluxes

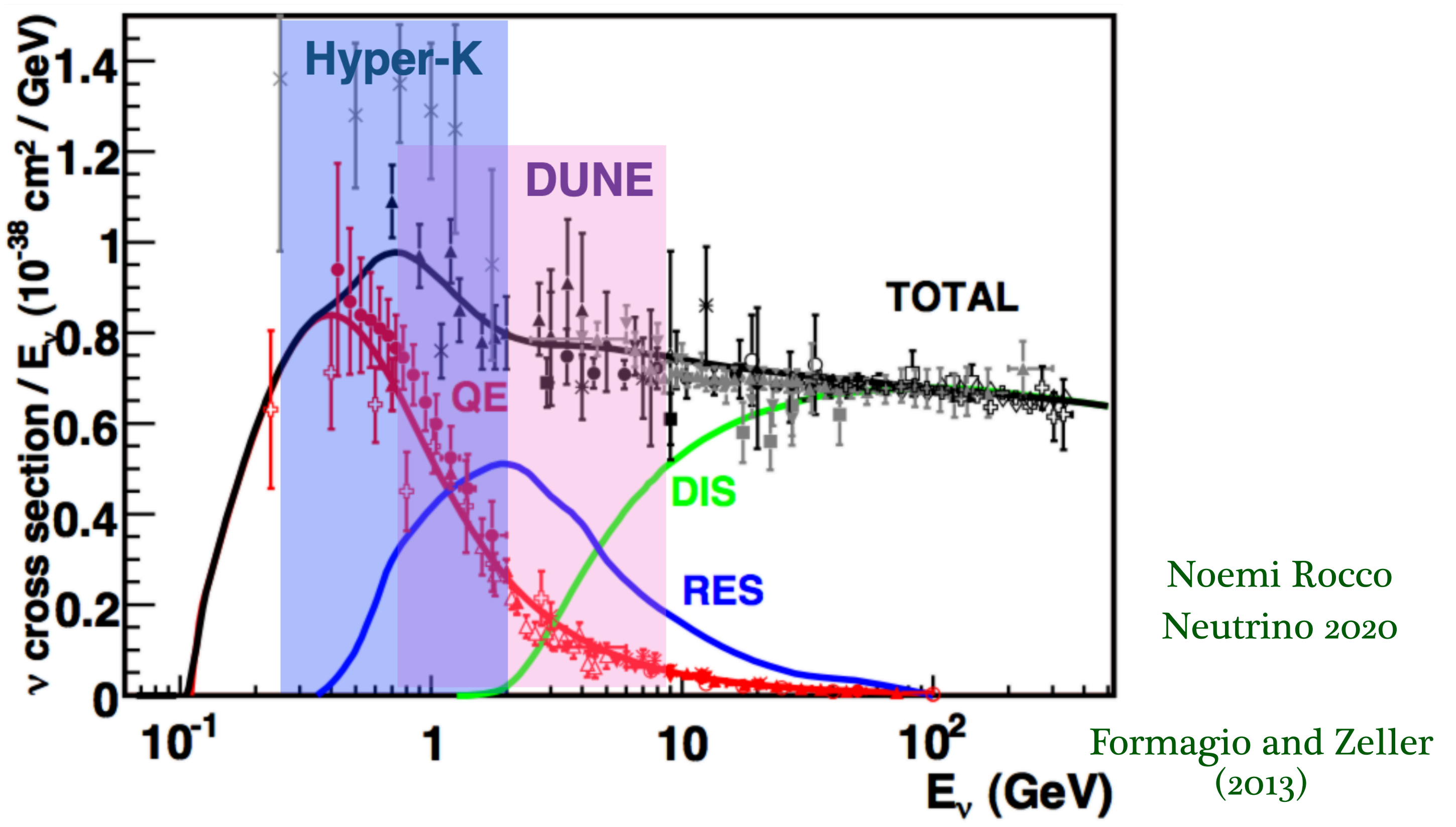

- basic process: bulk of events at Hyper-K and DUNE

- best channel for reconstruction of neutrino energy 


\section{CCQE scattering on free nucleon}

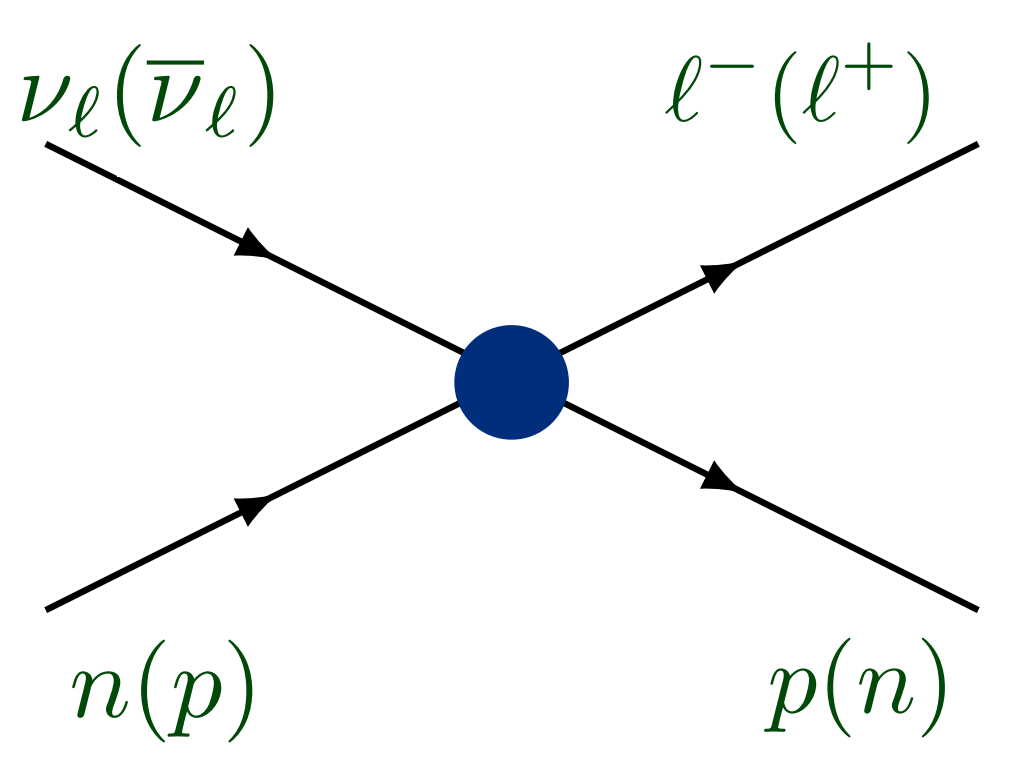

$$
\begin{gathered}
s-u=4 M E_{\nu}-Q^{2}-m_{\ell}^{2} \\
\tau=\frac{Q^{2}}{4 M^{2}}
\end{gathered}
$$

unpolarised observables are measured

cross section expression:

$$
\frac{\mathrm{d} \sigma}{\mathrm{d} Q^{2}} \sim \frac{M^{2}}{E_{\nu}^{2}}\left(A\left(Q^{2}\right) \frac{m_{\ell}^{2}+Q^{2}}{M^{2}}-B\left(Q^{2}\right) \frac{s-u}{M^{2}}+C\left(Q^{2}\right)\left(\frac{s-u}{M^{2}}\right)^{2}\right)
$$

- structure-dependent functions:

$$
\begin{aligned}
A= & 2 \tau\left(F_{D}^{V}+F_{P}^{V}\right)^{2}-(1+\tau)\left[\left(F_{D}^{V}\right)^{2}+\tau\left(F_{P}^{V}\right)^{2}-\left(F_{A}\right)^{2}\right] \\
& -\frac{m_{\ell}^{2}}{4 M^{2}}\left[\left(F_{D}^{V}+F_{P}^{V}\right)^{2}+\left(F_{A}+2 F_{P}\right)^{2}-4(1+\tau) F_{P}^{2}\right] \\
B= & \pm 4 \tau F_{A}\left(F_{D}^{V}+F_{P}^{V}\right) \quad C=\frac{1}{4}\left[\left(F_{D}^{V}\right)^{2}+\tau\left(F_{P}^{V}\right)^{2}+\left(F_{A}\right)^{2}\right]
\end{aligned}
$$

- cross section measurements give us axial form factor 


\section{CCQE scattering on free nucleon}

- only 3 experiments performed with deuterium bubble chamber direct access to form-factor shape

ANL 1982: 1737 events

BNL 1981: 1138 events

FNAL 1983: 362 events

world data: $\sim 3200$ events

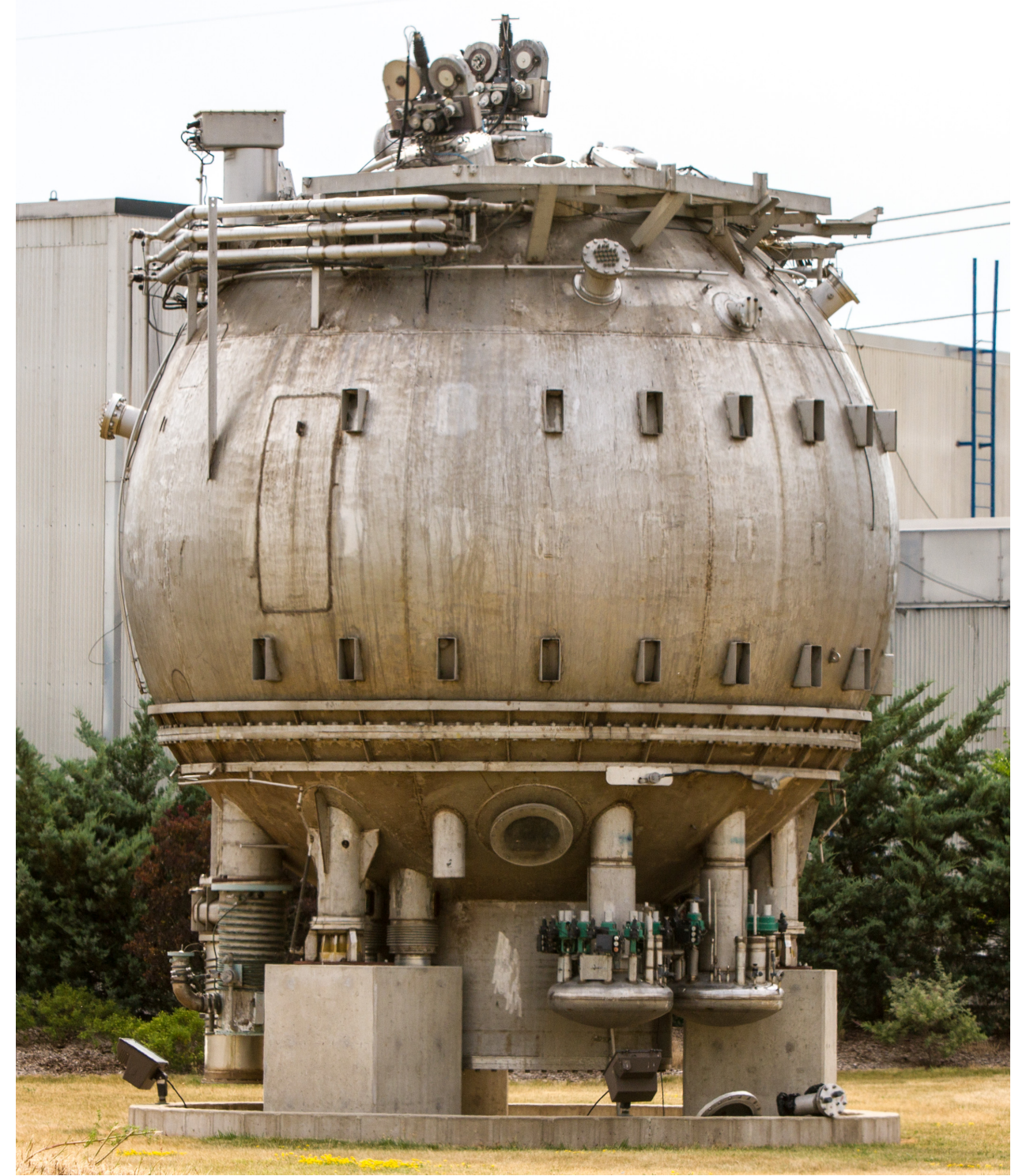

Fermilab bubble chamber, Richard Drew

- cross section measurements give us axial form factor 


\section{CCQE scattering cross section}

- dark band: uncertainty of iso 1 fit

- light band: uncertainty of axial form factor

- blue line: BBBA2005 fit of electromagnetic form factors

Hyper-K $\sigma_{v_{\mu}, \mu^{-p}}\left(E_{\nu}\right)$

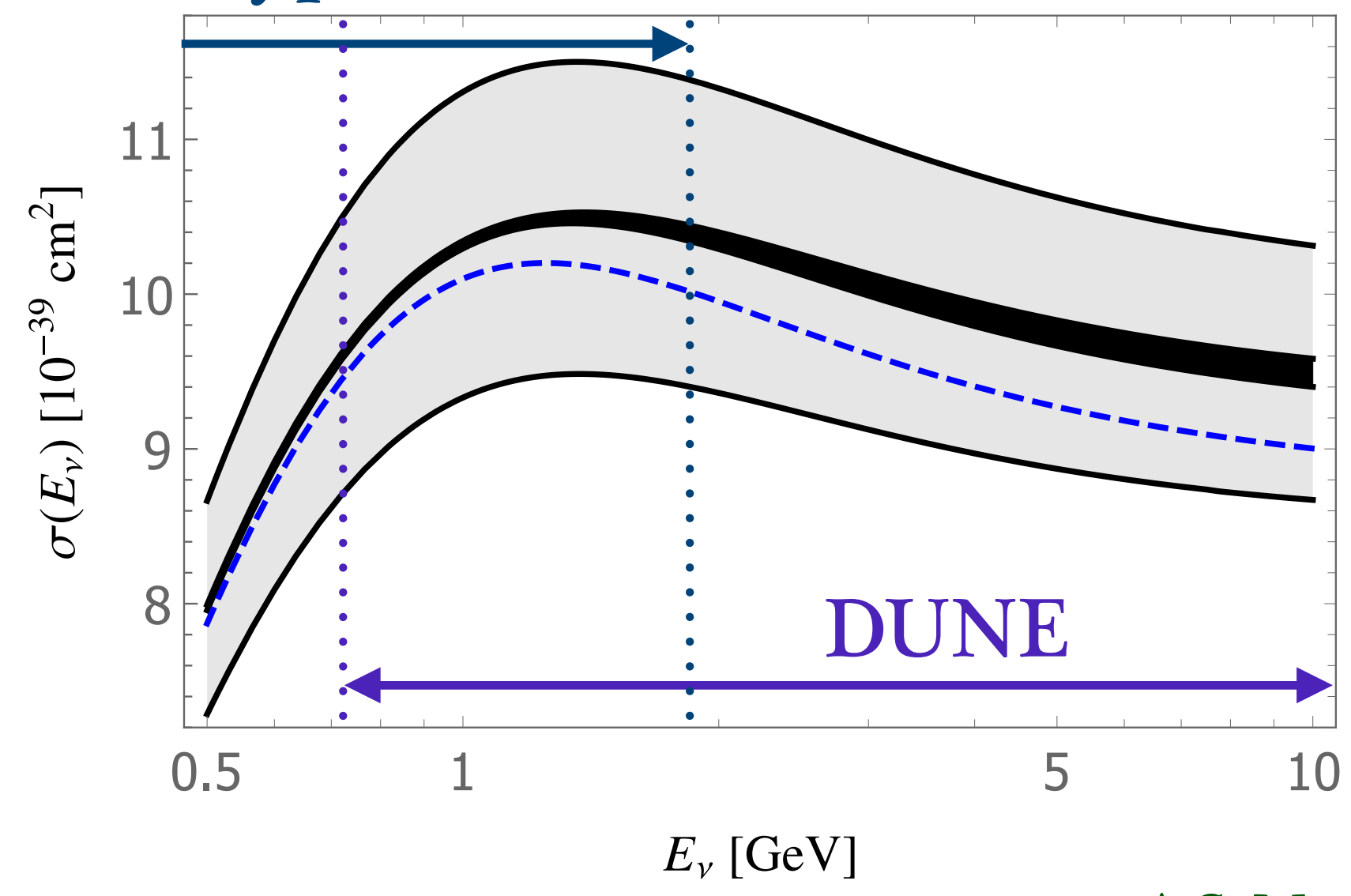

Hyper-K $\quad \sigma_{\bar{\nu}_{\mu} p \rightarrow \mu^{+} n}\left(E_{\bar{\nu}}\right)$

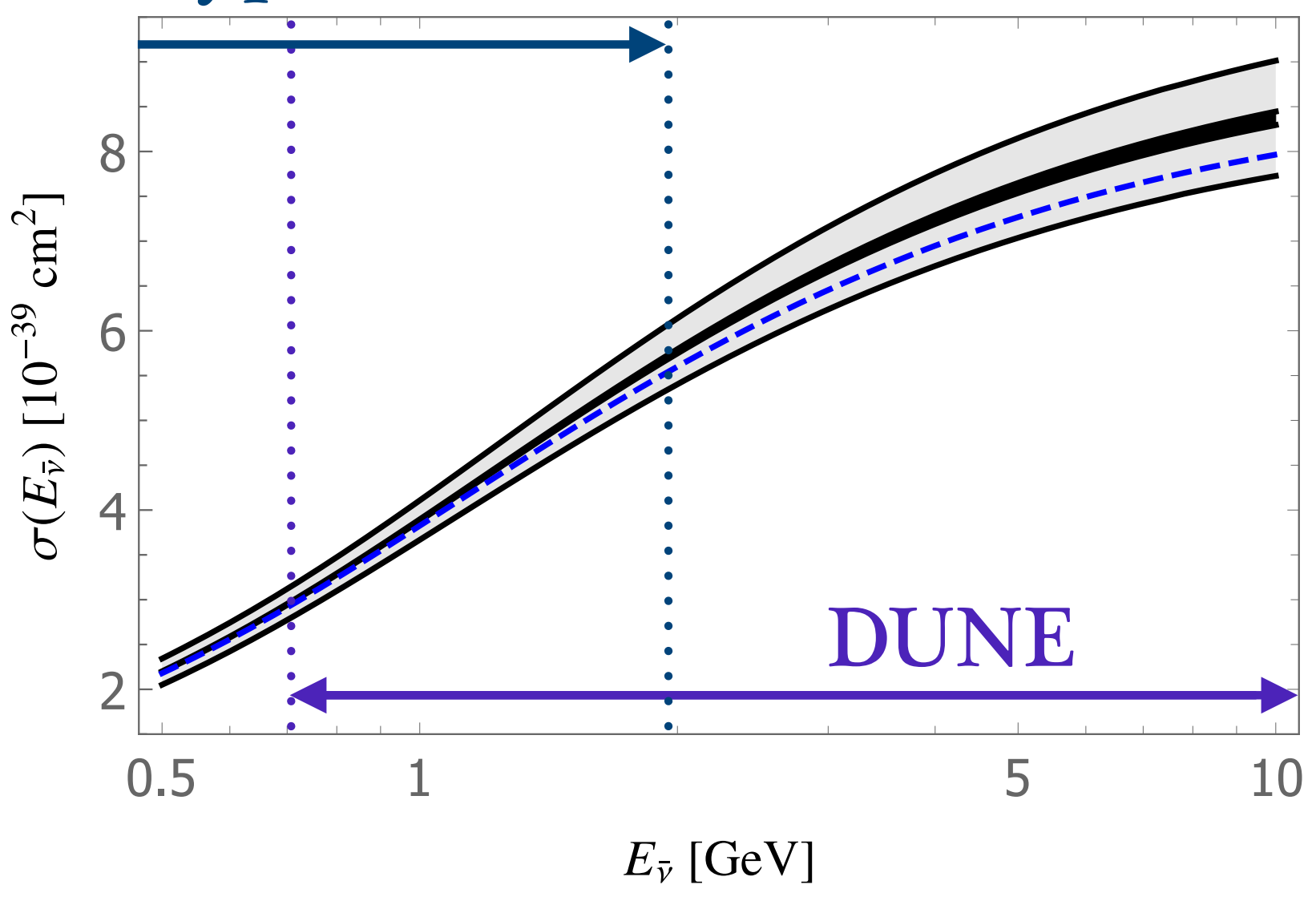

A.S. Meyer, M. Betancourt, R. Gran and R.J. Hill (2016)

Kaushik Borah, Richard J. Hill, Gabriel Lee and O. T. (arXiv: 2003.13640) 


\section{Form factors and polarization observables}

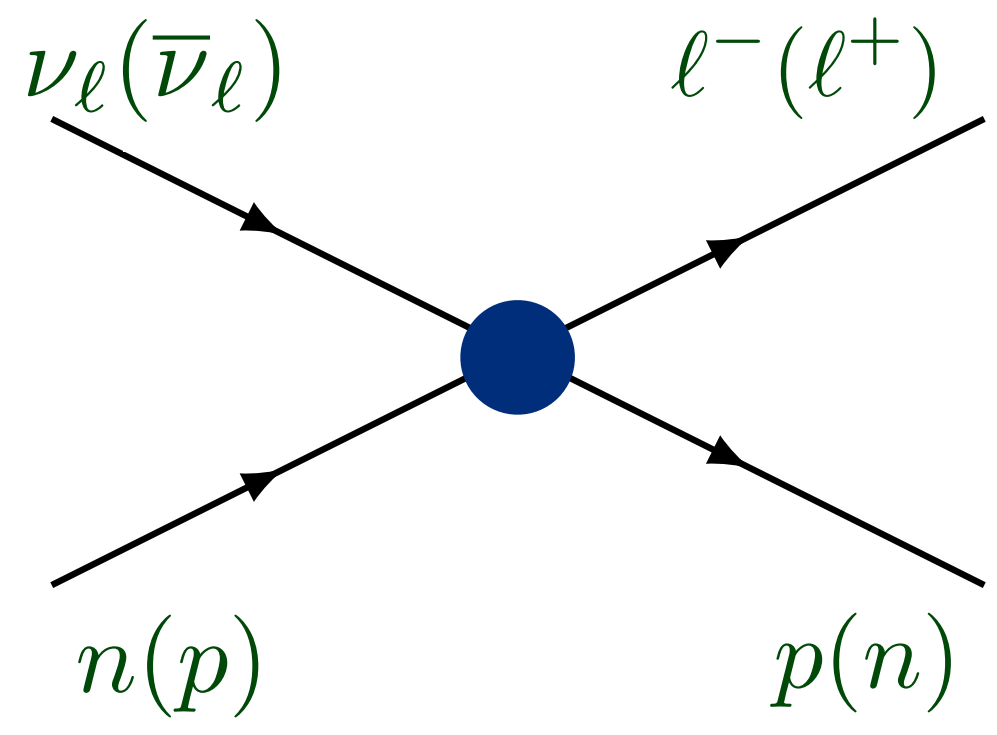

polarized target and/or recoil

$$
A=\frac{\mathrm{d} \sigma(S)-\mathrm{d} \sigma(-S)}{\mathrm{d} \sigma(S)+\mathrm{d} \sigma(-S)}
$$

- spin asymmetries are not suppressed by $m_{\ell}$ or coupling constant: comparable to unpolarized cross section rates

- sensitivity to axial form factor at $\mathrm{GeV}$ muon antineutrino energies recent studies: Samoil M. Bilenky and Ekaterina Christova (2013) Krzystof M. Graczyk and Beata E. Kowal (2019)

- sensitivity to pseudoscalar form factor with tau (anti)neutrino and muon (anti)neutrino of hundreds $\mathrm{MeV}$ energies

O. T. (arXiv: 2008.03527)

- pseudoscalar form factor can be accessed independently - sizable contribution of axial form factor at $\mathrm{GeV}$ energies 


\section{CCQE scattering cross section}

- dark band: uncertainty of iso 1 fit

- light band: uncertainty of axial form factor

- blue line: BBBA2005 fit of electromagnetic form factors

Hyper-K $\sigma_{v_{\mu}, \mu^{-p}}\left(E_{\nu}\right)$

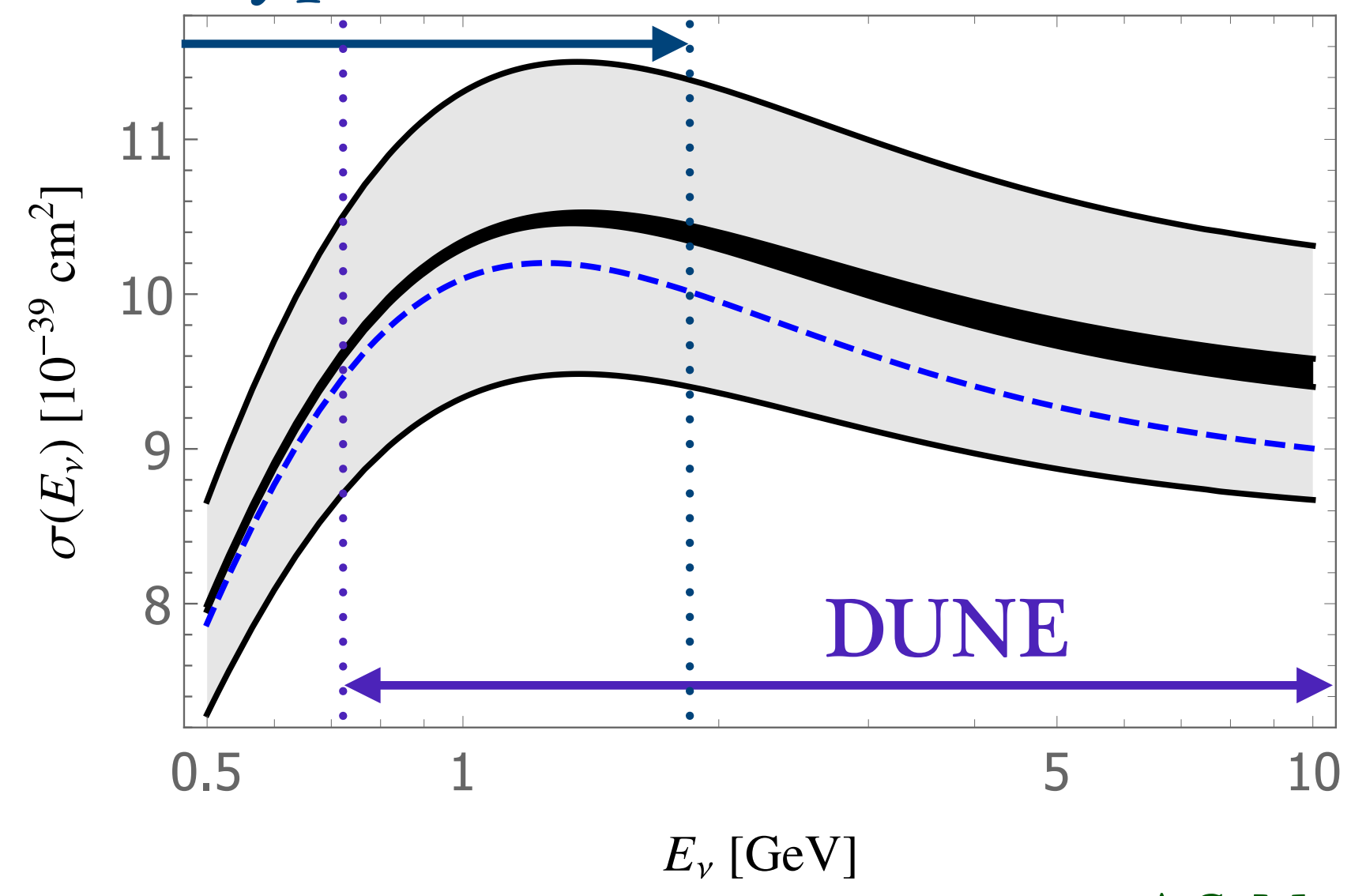

Hyper-K $\quad \sigma_{\bar{\nu}_{\mu} p \rightarrow \mu^{+} n}\left(E_{\bar{\nu}}\right)$

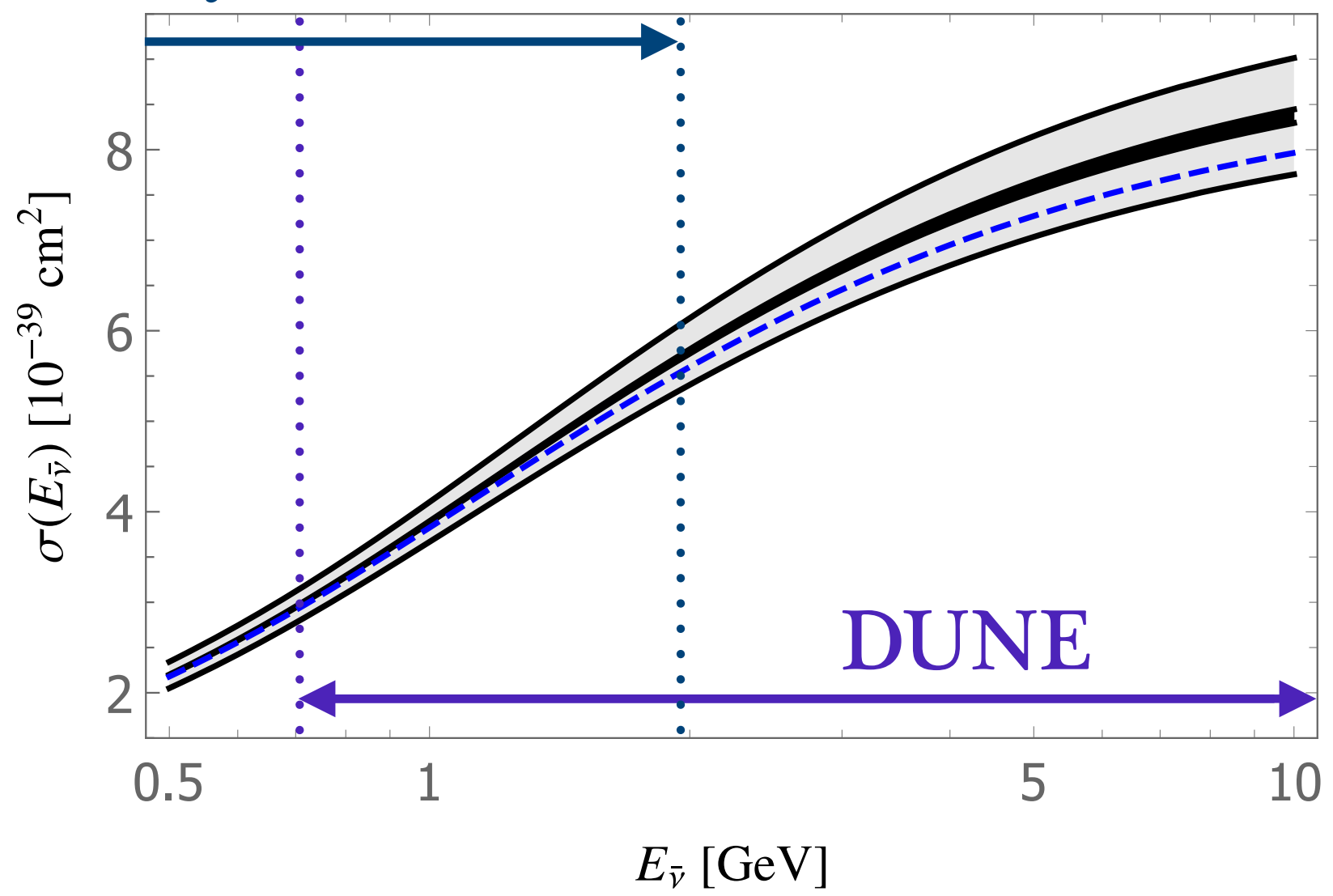

A.S. Meyer, M. Betancourt, R. Gran and R.J. Hill (2016)

Kaushik Borah, Richard J. Hill, Gabriel Lee and O. T. (arXiv: 2003.13640)

- strong motivation to reduce $10-20 \%$ error of axial form factor 


\section{$\underline{\text { Radiative corrections in CCQE }}$}

- large kinematical logarithms enhance radiative corrections

$$
\frac{\alpha}{\pi} \sim 0.2 \% \quad \text { multiplied by } \quad \ln \frac{E_{\nu}}{m_{\ell}} \sim 6-10
$$

A. De Rujula, R. Petronzio and A. Savoy-Navarro (1979) Melanie Day and Kevin S. McFarland (2012)

O. Tomalak and R.J. Hill (2020)

- CCQE with electron flavor is subject to large corrections

- phase-space restrictions enhance radiative corrections

$$
\begin{array}{lll}
\frac{\alpha}{\pi} \sim 0.2 \% & \text { multiplied by } & \ln ^{2} \frac{E_{\nu}}{m_{\ell}} \sim 36-100 \\
E_{\gamma}<\Delta E & \text { soft photons } & 4 \ln \frac{E_{\nu}}{m_{\ell}} \ln \frac{\Delta E}{m_{\ell}} \sim 70-120
\end{array}
$$

smaller collinear logarithms

- crucial dependence on detector details

- radiative corrections crucial for \%-level oscillation expts 


\section{Factorization approach}

- cross section with soft photons is given by factorization formula

$$
\mathrm{d} \sigma \sim S\left(\beta_{\ell}, \frac{\Delta E}{\mu}\right) J\left(\frac{m_{\ell}}{\mu}\right) H\left(\frac{M}{\mu}, \ldots\right)+\mathrm{O}\left(\frac{m_{\ell}^{2}}{M^{2}}, \frac{m_{\ell}^{2}}{M E_{\nu}}\right)
$$

- determine hard function at hard scale matching experiment or model to the theory with heavy nucleon 


\section{Interaction with nucleons}

- QCD running coupling

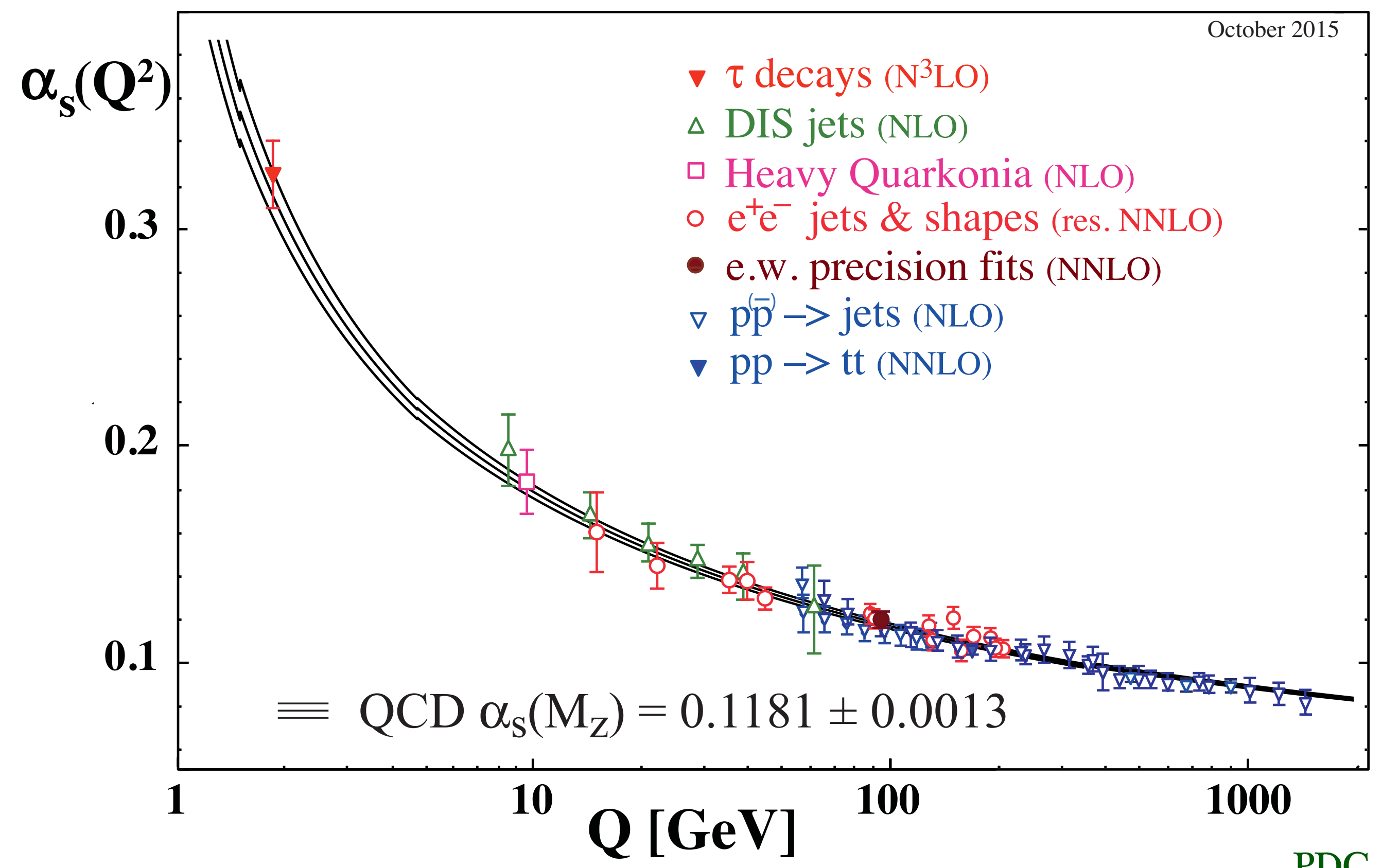

PDG 2015

- hadrons are correct degrees of freedom at GeV energy 


\section{Hadronic model at $\mathrm{GeV}$ scale}

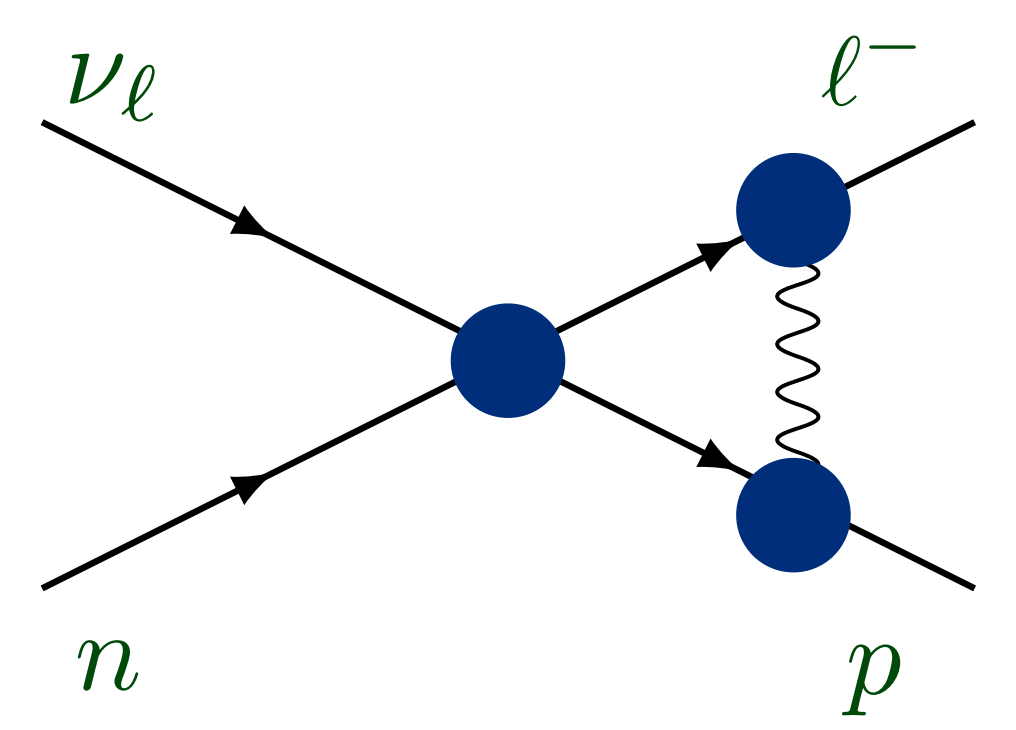

- exchange of photon between the charged lepton and proton

- assume onshell form for each interaction vertex

discussed for CCQE: Krzystof M. Graczyk (2013)

- add self energy for charged particles

- the best determination of hard function

- add real soft and collinear radiation on top

- correct kinematical and infrared logarithms 


\section{Factorization approach}

- cross section with soft photons is given by factorization formula

$$
\mathrm{d} \sigma \sim S\left(\beta_{\ell}, \frac{\Delta E}{\mu}\right) J\left(\frac{m_{\ell}}{\mu}\right) H\left(\frac{M}{\mu}, \ldots\right)+\mathrm{O}\left(\frac{m_{\ell}^{2}}{M^{2}}, \frac{m_{\ell}^{2}}{M E_{\nu}}\right)
$$

- determine hard function at hard scale matching experiment or model to the theory with heavy nucleon

- RGE evolution of the hard function to scales $\Delta E, m_{\ell}$

$+\Delta E-$ soft and collinear functions are evaluated perturbatively

- calculate cross section at low energies accounting for all large logs

ep scattering: R.J. Hill (2016)

- soft and collinear functions obtained analytically

- hard function describes physics at $\mathrm{GeV}$ energies 


\section{Cross section. Preliminary results}
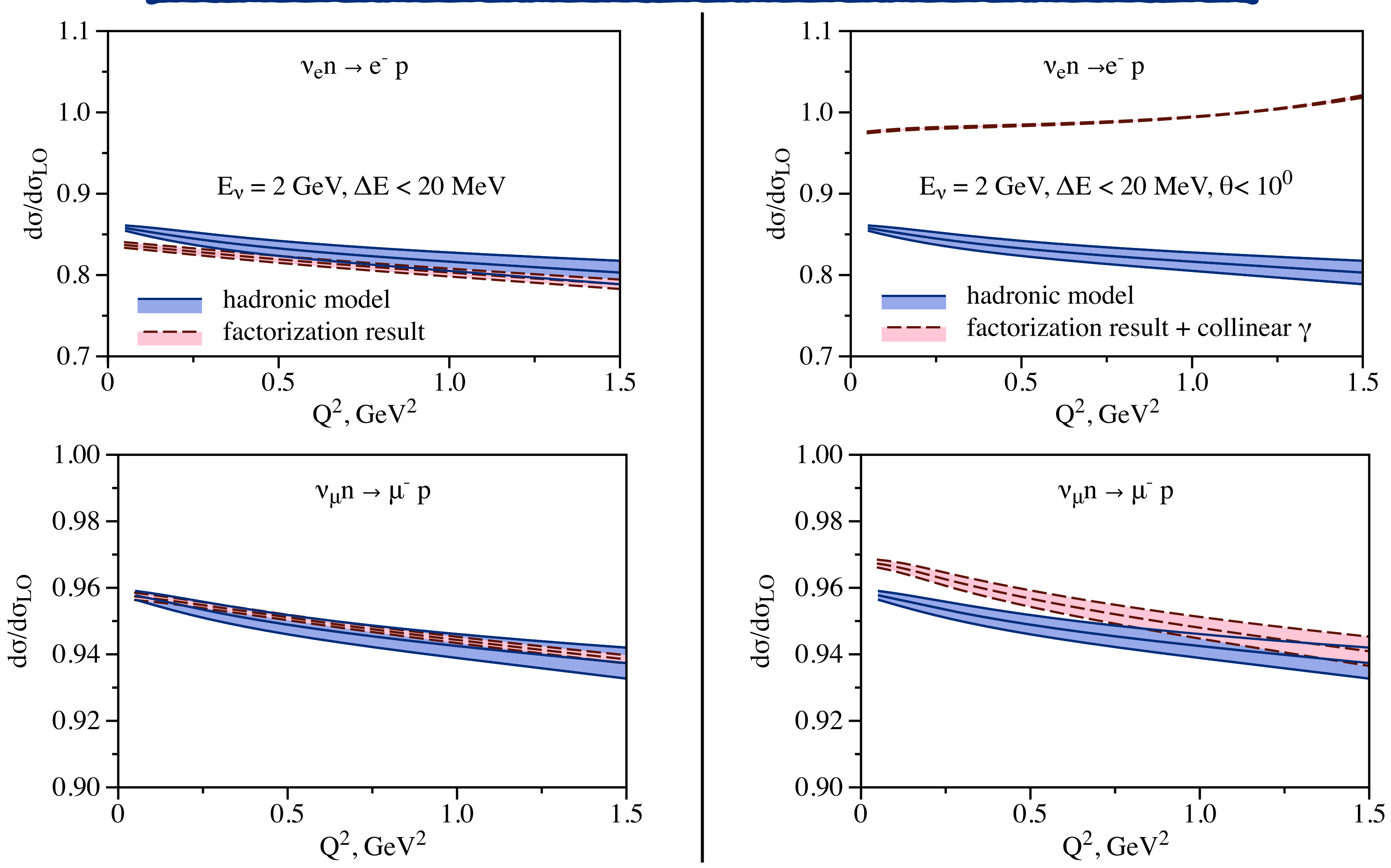

- corrections with e larger; cancellation virtual vs real 


\section{Cross section. Preliminary results}
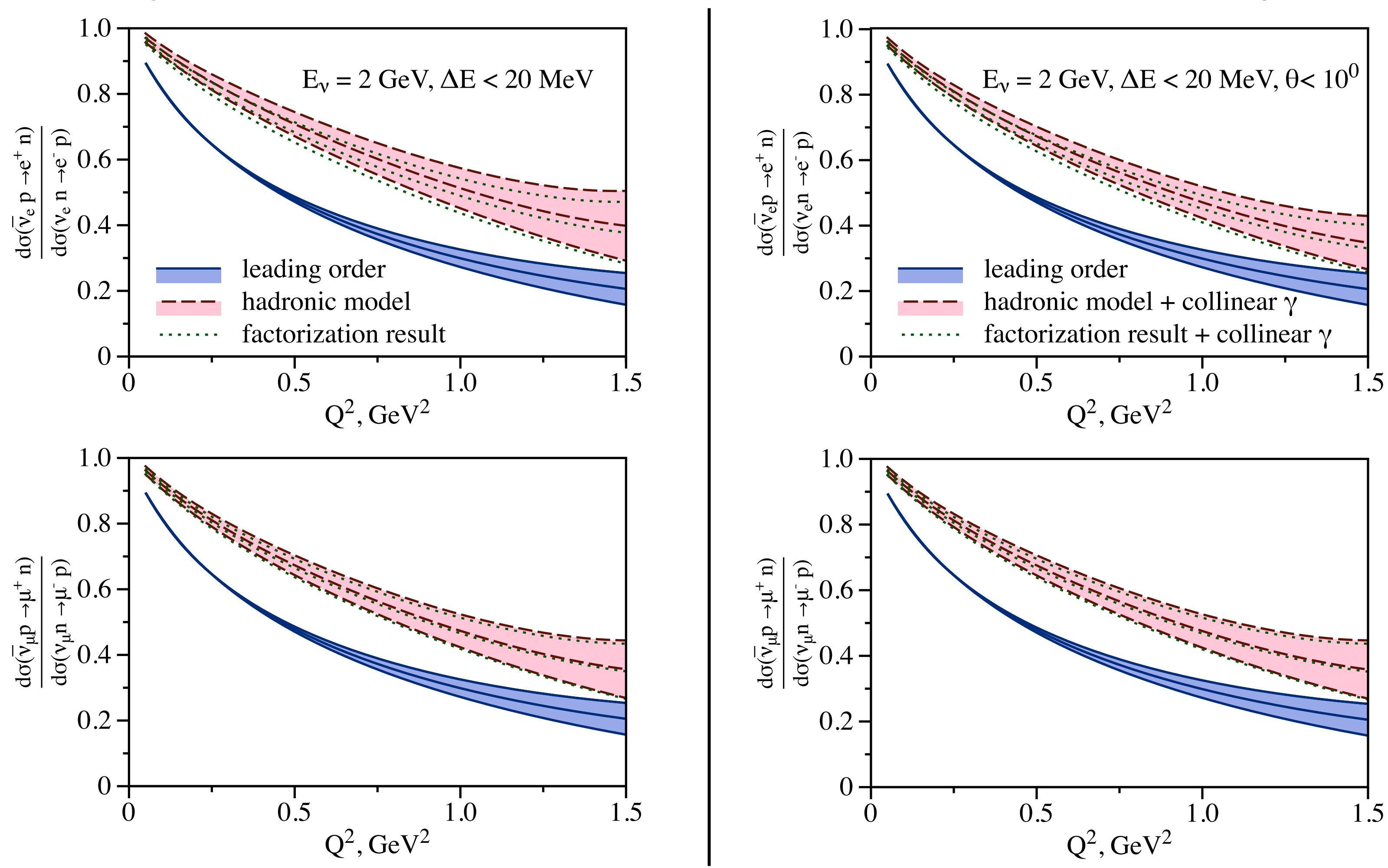

- similar muon and electron anti- over neutrino ratios 


\section{Cross section. Preliminary results}
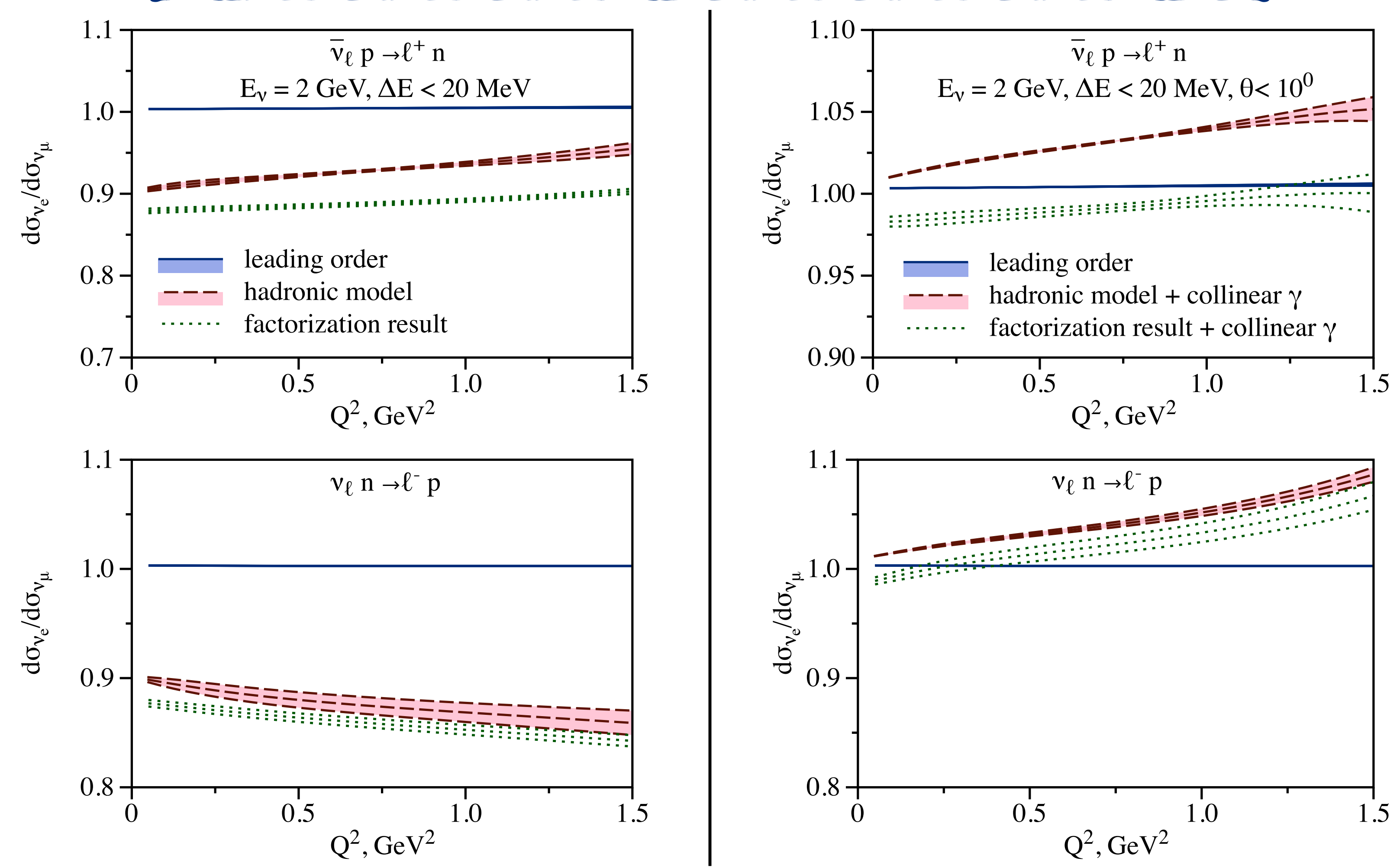

- similar muon and electron cross sections with collinear $\gamma$ 


\section{Conclusions}
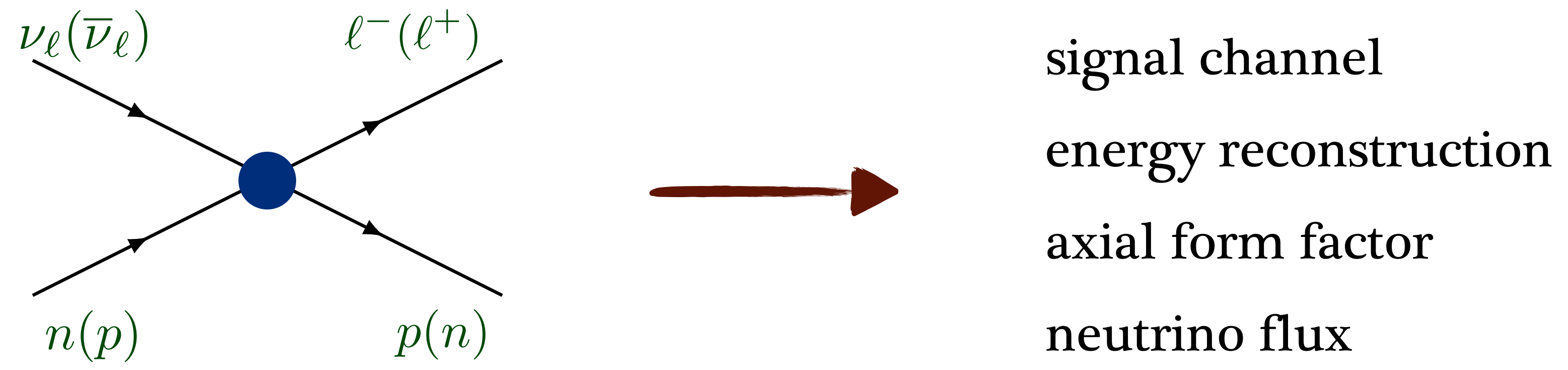

- radiative corrections are formulated in factorization framework

- model for hard function: hadronic model with nucleon state

- kinematical, phase-space and perturbative logarithms included

- radiative corrections are calculated with \% level of accuracy

\section{Ongoing}

- improve error estimate

- quantify details of real radiation 
Thanks for your attention !!! 